\title{
Barrow-in-Furness: a large community legionellosis outbreak in the UK
}

\author{
E. BENNETT ${ }^{1} *$, M. ASHTON ${ }^{2}$, N. CALVERT ${ }^{3}$, J. CHALONER ${ }^{4}$, \\ J. CHEESBROUGH ${ }^{5}$, J. EGAN ${ }^{1}$, I. FARRELL ${ }^{6}$, I. HALL ${ }^{1}$, T. G. HARRISON ${ }^{7}$, \\ F. C. NAIK ${ }^{8}$, S. PARTRIDGE ${ }^{9}$, Q. SYED ${ }^{10}$ AND R. N. GENT ${ }^{1}$ \\ ${ }^{1}$ Emergency Response Department, Public Health England, Salisbury, UK \\ ${ }^{2}$ Knowsley Metropolitan Borough Council, Knowsley, Merseyside, UK \\ ${ }^{3}$ Public Health England, Penrith, Cumbria, UK \\ ${ }^{4}$ NHS Fylde and Wyre, Fylde and Wyre, UK \\ ${ }^{5}$ Lancashire Teaching Hospitals, NHS Foundation Trust, Lancashire, UK \\ ${ }^{6}$ North West Regional Microbiologist, Health Protection Agency, Warrington, UK \\ ${ }_{8}^{7}$ Microbiology Reference Services, Public Health England, London, UK \\ ${ }^{8}$ Centre for Infectious Disease Surveillance and Control (CIDSC), Public Health England, London, UK \\ ${ }^{9}$ West Suffolk NHS Foundation Trust, Suffolk, UK \\ ${ }^{10}$ Public Health England, Cheshire and Merseyside, UK
}

Received 29 April 2013; Final revision 4 September 2013; Accepted 6 September 2013; first published online 11 October 2013

\section{SUMMARY}

A community outbreak of legionellosis occurred in Barrow-in-Furness, Cumbria, during July and August 2002. A descriptive study and active case-finding were instigated and all known wet cooling systems and other potential sources were investigated. Genotypic and phenotypic analysis, and amplified fragment length polymorphism of clinical human and environmental isolates confirmed the air-conditioning unit of a council-owned arts and leisure centre to be the source of infection. Subsequent sequence-based typing confirmed this link. One hundred and seventy-nine cases, including seven deaths [case fatality rate (CFR) 3.9\%] were attributed to the outbreak. Timely recognition and management of the incident very likely led to the low CFR compared to other outbreaks. The outbreak highlights the responsibility associated with managing an aerosol-producing system, with the potential to expose and infect a large proportion of the local population and the consequent legal ramifications and human cost.

Key words: Barrow-in-Furness, cooling tower, community outbreak, Legionnaires' disease, legionellosis.

\section{INTRODUCTION}

Legionellosis describes the pneumonic [Legionnaires' disease (LD)] and non-pneumonic forms of infection caused by the Legionella bacteria. People become

\footnotetext{
* Author for correspondence: Mrs E. Bennett, Health Protection Agency, Microbial Risk Assessment, Centre for Emergency Preparedness and Response, Salisbury SP4 0JG, UK. (Email: emma.bennett@phe.gov.uk)
}

infected when they inhale air carrying the bacteria in aerosolized form from a contaminated source. Deaths from LD occur in $10-15 \%$ of the general population, but may be higher in immunocompromised patients. In recent large community outbreaks, overall case fatality rates (CFRs) have been about $10 \%$ (range $0-32 \%$ ) and $\sim 70 \%$ of $\mathrm{LD}$ cases have required hospitalization (range $\sim 40 \%$ to $\sim 90 \%$ ) [1-4]. The incubation period can range from 2 to 19 days (median of 6-7 days after exposure) [3]. 
Legionella bacteria are found widely in the environment in both natural and artificial water sources, such as water towers associated with cooling systems, hot and cold water systems and spa pools. The bacteria only become a risk to human health when the ambient temperature is between $20^{\circ} \mathrm{C}$ and $46^{\circ} \mathrm{C}$ and nutrients (e.g. algae and amoebae) are available to promote active multiplication of the organism, such as in water systems which are not properly designed, installed and/or maintained. Control and prevention of the disease is through treatment of the source of the infection and by ensuring good system design and maintenance to prevent bacterial growth.

This paper describes a legionellosis outbreak that occurred during July and August 2002, in Barrowin-Furness (Barrow), a small industrial town in Cumbria, North West England. Following reports of a significant increase in local admissions with community-acquired pneumonia to Furness General Hospital (FGH), the first LD case was identified on 30 July (confirmed 31 July). A second case was identified on 1 August (confirmed 2 August) and, when both cases were epidemiologically linked, an outbreak control team (OCT) was convened. Over 550 premises in the Barrow area were identified as potential sources, and in these risk assessments and sampling were carried out. One of these premises, an air-conditioning unit of a council-owned Arts and Leisure centre, Forum 28, was identified as a possible source of infection and pre-emptively shut down the day before the OCT was convened. Descriptive epidemiology and microbiological analyses confirmed the airconditioning unit within this facility as the source of the outbreak. In total, 179 cases of legionellosis (145 cases of LD and 34 cases of non-pneumonic legionellosis) were confirmed to have resulted from Legionella pneumophila serogroup 1 infection, and of these, seven cases died.

Managing a large community legionellosis outbreak is inherently demanding, due to the often protracted nature of the events and the requirement for a prolonged response. Many challenges were met through the introduction of a novel approach to investigating the outbreak [5, 6]. For the first time, the Police and Health and Safety Executive (HSE) ran parallel criminal and forensic investigations alongside the outbreak investigation team. The success of this approach signalled a new phase in public health response in the UK, a result of which saw charges brought against an organization. The outbreak witnessed the first major incident to be handled by the emerging Health Protection Agency, an independent UK public health organization set up by the Government in 2003 [now part of Public Health England (https://www.gov.uk/government/organisations/publichealth-england)]. Specifically, in the North West of England, this collaborative approach between public health, environmental health, local National Health Service (NHS) and the HSE resulted in the successful resolution of the largest legionellosis outbreak experienced in the UK to date.

\section{METHODS}

\section{Case-finding and epidemiology}

Following the convening of the OCT, active casefinding was initiated; suspected cases were assessed in the admitting Accident and Emergency Department and in-patients reviewed. In the weeks after the outbreak, chest X-rays were re-evaluated and detailed patient assessments carried out at follow-up clinics. Proactive communication was adopted from the outset in an effort to support the local population, with health professionals and the media being kept up-to-date with the unfolding investigation. Within a day of the first OCT meeting a national alert was sent out and guidelines were cascaded, by letter, to all General Practioners (GPs) and casualty departments in the country, to help identify possible cases and to provide clinical management advice. Details were circulated to all Consultants in Communicable Disease Control (CCDCs) in the North West, and updates were regularly published on Cumbria and Lancashire Health Protection Unit's website as well as being provided to GPs. Regular press releases and press conferences were made, the first taking place on 3 August, and timely journal articles were published to increase and maintain awareness $[7,8]$ and a telephone helpline service was promptly established to assist with public enquiries. A full-time press officer was employed to deal specifically with this outbreak. More widely, a European alert was sent out on 1 August to all European Working Group for Legionella Infections (EWGLI) collaborators and the World Health Organization (WHO).

National surveillance scheme case definition criteria were used for the outbreak case definition for LD, plus an additional criterion, i.e. 'had visited the centre of Barrow between 1 and 31 July 2002'. A descriptive study was performed to collect data on exposure history and links with Barrow before the onset of illness, 


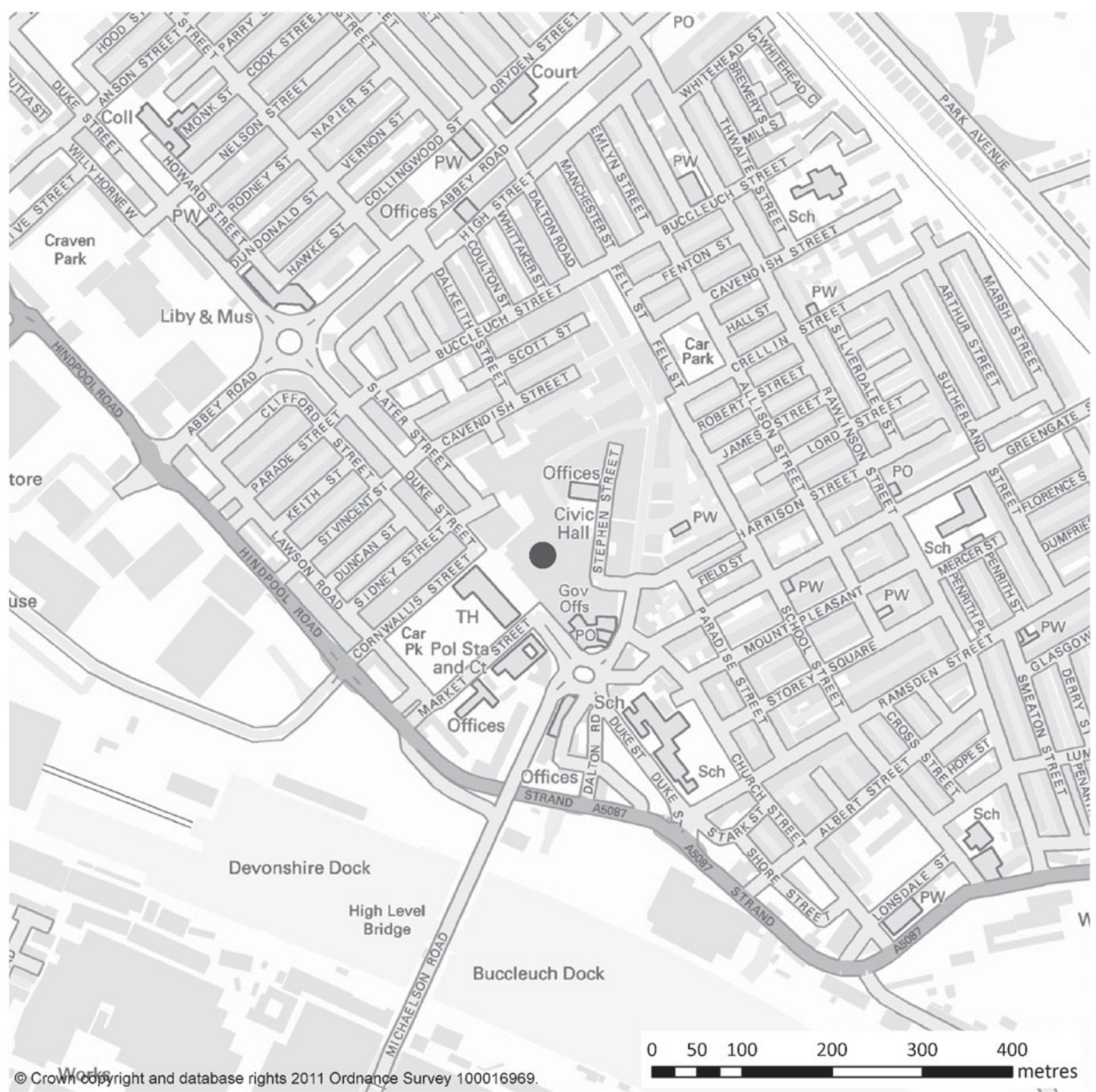

Fig. 1. Location of Forum 28 Arts and Leisure Centre, Barrow-in-Furness.

using the standard national enhanced surveillance form and an additional page for movement details in Barrow town centre. Interviews were conducted face-to-face in hospital or in a follow-up clinic and, exceptionally, by telephone. A case-control study was considered, but not performed, since there was sufficient epidemiological, microbiological and environmental evidence early on indicating that the source of infection might be Forum 28. Final case numbers were determined through the analysis of serial blood tests, undertaken for many months after the outbreak was declared over.

\section{Environmental investigation}

Epidemiological linkage to Barrow was made between the first few reported cases in conjunction with a report from a senior Environmental Health Officer (EHO). The EHO reported large amounts of aerosol and water droplets emerging from an air-conditioning vent from Forum 28 into the alleyway (lane) (Figs 1 and 2), which instigated the survey and sampling of hot and cold water systems at Forum 28. To ensure that this probable source had been correctly implicated, the OCT (including Health Protection, 


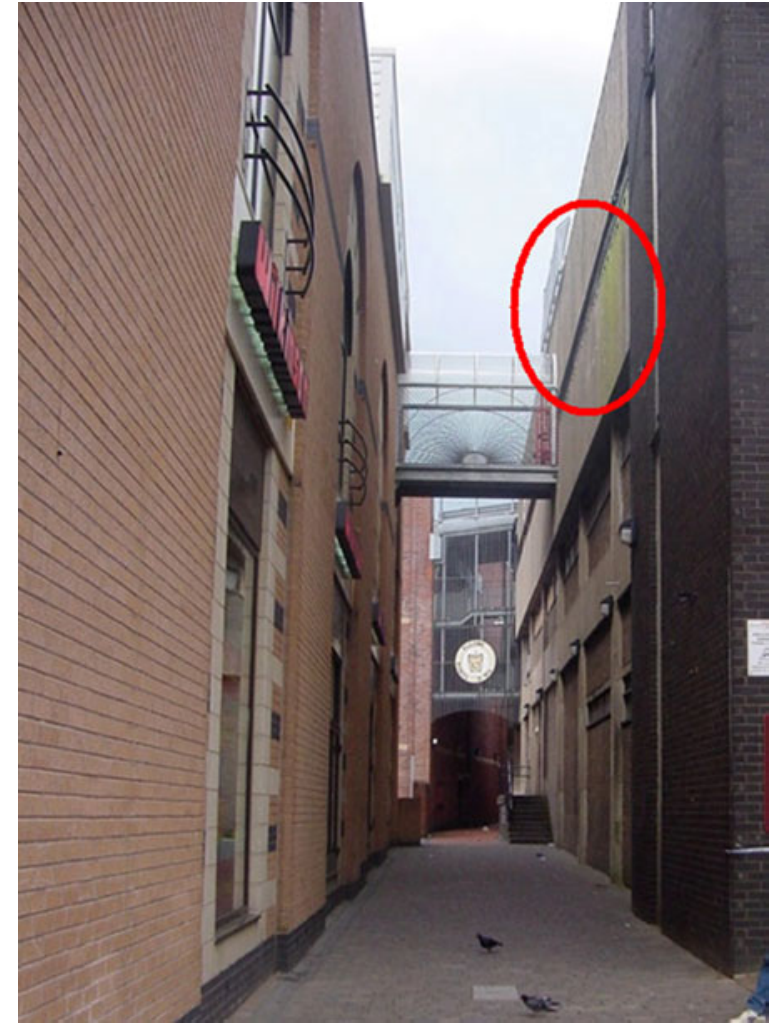

Fig. 2. Contemporary photograph of alleyway outside Forum 28 into which droplets of contaminated water were emitted. Red circle indicates the vent from which the Legionella-contaminated aerosol came. (Copyright (C) Dr Nigel Calvert, NHS Dumfries \& Galloway.)

Environmental Health, HSE, and public health teams) inspected every other potentially hazardous installation in the town, registered on the HSE and the local authority (LA) databases. Maintenance records were examined and samples of pooled or retained water obtained. Visual searches were conducted to identify any unregistered potential sources (UK businesses are required to register cooling towers with the relevant local government authority). Local nursing and residential homes, where two cases had lived, were also sampled.

\section{Microbiological investigation}

Urine samples from suspected cases were tested, on hospital admission, using commercially available $L$. pneumophila urinary antigen (UAg) kits (e.g. BinaxNow ICT, Alere, USA) and confirmed by the Respiratory and Systemic Infection Laboratory (RSIL), using their in-house enzyme-linked immunoassay (EIA) specific for a subset [designated monoclonal antibody positive $(\mathrm{mAb} 2+\mathrm{ve})]$ of $L$. pneumophila serogroup 1 strains [9]. Wherever a respiratory sample was obtained, isolation of L. pneumophila was attempted using standard culture techniques [10]. Isolates were characterized by monoclonal antibody subgrouping [11] and amplified fragment-length polymorphism (AFLP) typing [12]. Subsequently, isolates were further examined using DNA sequence-based typing (SBT) $[13,14]$. However, more often sera was obtained and examined by indirect immunofluorescent antibody assay (IFAT) using formalized yolk-sac antigen (FYSA), as previously described [15]. Environmental water samples were collected and processed by standard methods [16] and water concentrates were examined by direct immunofluorescence assay (DFA) as previously described for clinical samples [17].

\section{Police investigation}

A parallel criminal and forensic investigation was led by Barrow Division of Cumbria Police and the HSE to examine possible breaches under health and safety law. Sites under a high degree of suspicion, or with particular evidential significance, were surveyed and sampled by Health Protection Unit or Public Health Laboratory Service (PHLS) staff, in the presence of police scene-of-crime officers. These samples were submitted for testing to PHLS under chain of evidence controls.

\section{RESULTS}

\section{Descriptive epidemiology}

Despite living nearly 100 miles apart, the first two cases of legionellosis were rapidly epidemiologically linked from their activity/14-day history diaries, and an OCT convened the following day. In total, 2579 persons were evaluated, of which 494 were clinically diagnosed as possible cases of legionellosis and admitted to hospital. Of these, 220 were actively treated as presumptive cases (Fig. 3, Table 1); 179 of which fulfilled the case definitions for legionellosis and were included in the analysis. The primary microbiological method of diagnosis was made by culture for 16 cases; UAg detection in 134 cases and serological testing in 29 cases (Fig. 4). A further 15 (of the 220) cases had equivocal serology results, i.e. low level seroconversion and therefore did not fulfil the case definition and were excluded from the analysis. One hundred and forty-five cases $(81 \%)$ were confirmed as LD with evidence of pneumonia and 34 cases were confirmed as legionellosis without evidence of pneumonia (Fig. 4). During the acute outbreak 
Table 1. Outbreak case definitions used for the Barrow-in-Furness outbreak, 2002

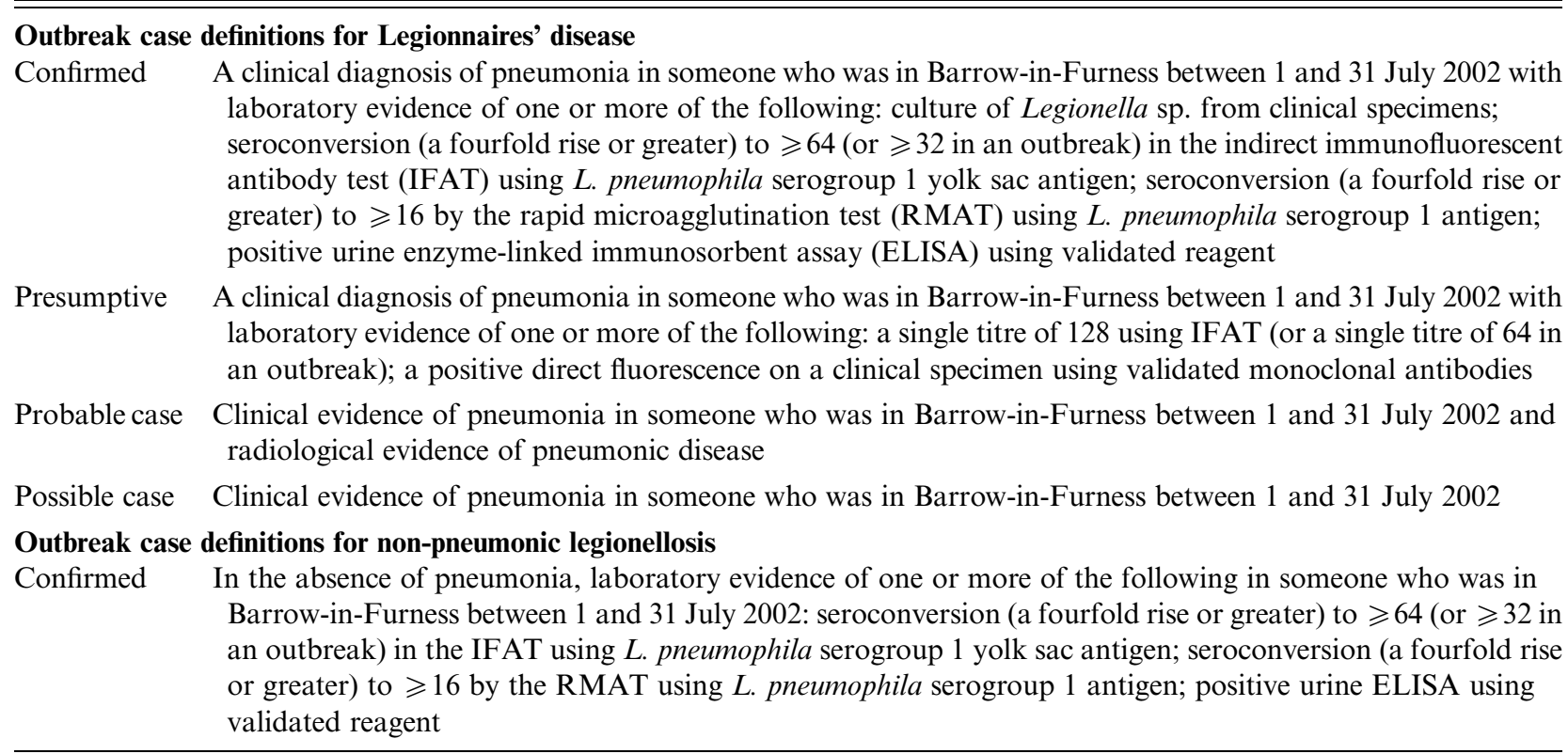

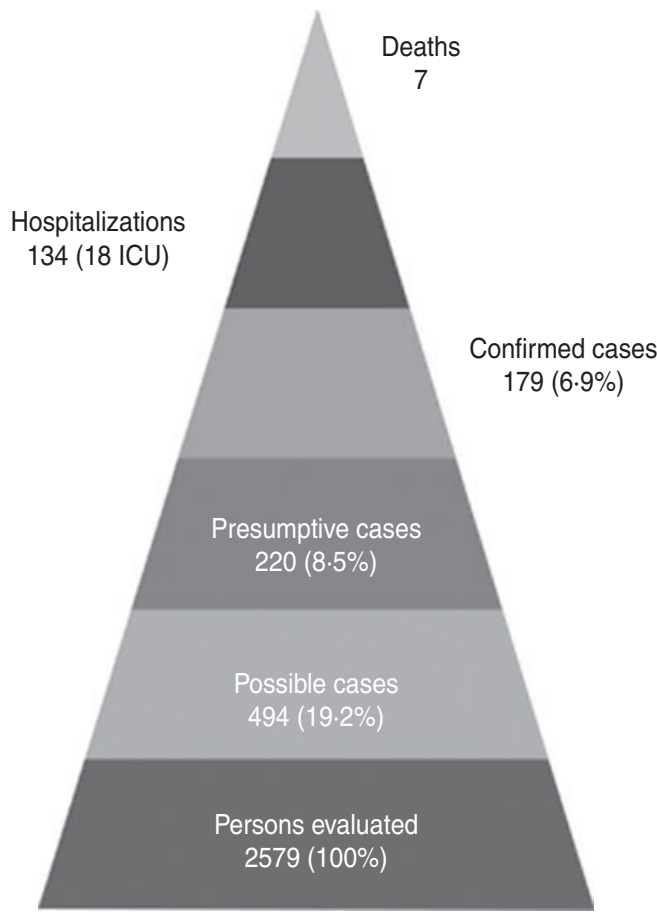

Fig. 3. Disease burden during the outbreak.

phase, the coroner recorded seven deaths (six female) in patients associated with the outbreak-five as a direct result of $L$. pneumonia infection, and two where LD was a contributing factor. All deaths were LD cases (three culture positive, four UAg positive), giving a CFR of $3.9 \%$ for all cases $(4 \cdot 8 \%$ for pneumonic LD cases). Four were admitted to intensive care units, two were on general medical wards (one diagnosed at post mortem) and one case died at home. Of note, the coroner suggested that a number of other deaths in convalescent patients may have been precipitated by infection with legionellosis.

A wide age distribution was observed ranging from 23 to 102 years. The mean (and median) age of all legionellosis cases was 63 years, and was not significantly different between the sexes $(P=0.63) ; 63.6$ years for the $82(46 \%)$ females and 62.6 years for the $97(54 \%)$ males (Table 1). The mean age of pneumonic cases was borderline significantly older than the mean age of non-pneumonic cases (64 vs. $59 \cdot 5$ years, $P=$ $0 \cdot 056)$. Of the 179 cases, $33(18 \%)$ were aged between 41 and 50 years, nine (5\%) were aged $31-40$ years and three were in their twenties. The attack rate for all ages was 250/100000 resident Barrow population (280 and $220 / 100000$ in males and females, respectively) and rose with increasing age (Table 2). Attack rates by home location are shown in Figure 5.

It is well known that comorbidities, such as diabetes, smoking and heavy alcohol consumption, are associated with an increased risk of legionellosis [18]; however, risk factor details were not recorded for the majority of this dataset, thus it is not possible to report any practical findings.

One hundred and thirty-four $(74.9 \%)$ cases required hospitalization; however, the admission status of nine cases that lived or were diagnosed outside the North West region was not known. Patients who were 
Table 2. Legionellosis cases and attack rates by age and sex per 100000 Barrow-in-Furness population

\begin{tabular}{|c|c|c|c|c|c|c|c|c|c|}
\hline \multirow[b]{2}{*}{$\begin{array}{l}\text { Age group } \\
\text { (years) }\end{array}$} & \multicolumn{3}{|c|}{ Males } & \multicolumn{3}{|c|}{ Females } & \multicolumn{3}{|c|}{ Total } \\
\hline & No. & $\%$ & $\begin{array}{l}\text { Attack rate } \\
/ 100000\end{array}$ & No. & $\%$ & $\begin{array}{l}\text { Attack rate } \\
/ 100000\end{array}$ & No. & $\%$ & $\begin{array}{l}\text { Attack rate } \\
/ 100000\end{array}$ \\
\hline$<20$ & 0 & 0 & 0 & 0 & 0 & 0 & 0 & 0 & 0 \\
\hline 20-29 & 2 & $2 \cdot 1$ & 50 & 1 & $1 \cdot 2$ & 030 & 3 & $1 \cdot 7$ & 040 \\
\hline $30-39$ & 3 & $3 \cdot 1$ & 60 & 2 & $2 \cdot 4$ & 040 & 5 & $2 \cdot 8$ & 050 \\
\hline $40-49$ & 12 & $12 \cdot 4$ & 250 & 10 & $12 \cdot 2$ & 210 & 22 & $12 \cdot 3$ & 230 \\
\hline 50-59 & 19 & $19 \cdot 6$ & 380 & 20 & $24 \cdot 4$ & 420 & 39 & $21 \cdot 8$ & 400 \\
\hline 60-69 & 29 & $29 \cdot 9$ & 780 & 15 & $18 \cdot 3$ & 410 & 44 & $24 \cdot 6$ & 590 \\
\hline 70-79 & 23 & $23 \cdot 7$ & 1010 & 22 & $26 \cdot 8$ & 710 & 45 & $25 \cdot 4$ & 840 \\
\hline$\geqslant 80$ & 9 & $9 \cdot 3$ & 899 & 12 & $14 \cdot 6$ & 521 & 21 & $11 \cdot 7$ & 635 \\
\hline Total & 97 & 100 & 280 & 82 & 100 & 220 & 179 & 100 & 250 \\
\hline
\end{tabular}

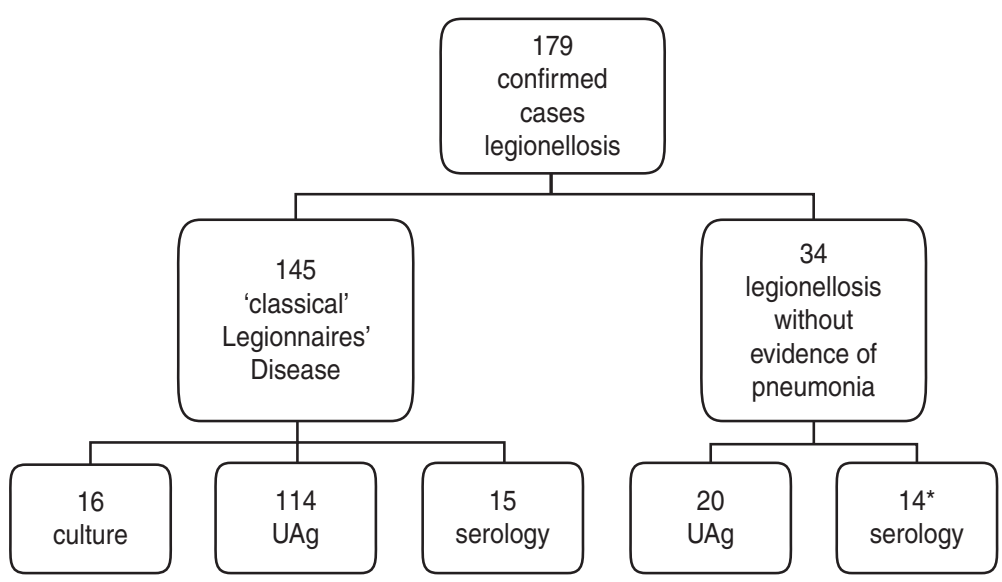

Fig. 4. Method of diagnosis for legionellosis cases reporting most reliable method. * Two cases accepted as legionellosis with clear conversion to 32 from negative.

hospitalized were significantly older than those not admitted (64.6 vs. 58.6 years, $P=0 \cdot 02)$. Eighteen $(10 \%)$ hospitalized cases received intensive care and/or ventilation in six intensive care units in hospitals around the counties of Cumbria and Lancashire. The more seriously ill cases required complex treatment regimens, particularly due to organ failure as a consequence of the disease. The mean age of fatal cases was 66 years, which was not significantly different to that of non-fatal cases (63 years, $P=0 \cdot 54$ ).

As a routine part of an outbreak investigation, efforts were made to obtain an activity diary/14-day history for each case. No other common locations were revealed from cases' travel histories other than their exposure to within $500 \mathrm{~m}$ of Forum 28 during the dates investigated. Of 142 cases where detailed diaries were clearly recorded, $42 \%$ reported visiting the town centre and frequently used the alleyway into which the air conditioning unit vented (Fig. 2), while $58 \%$ of cases visited the town centre only.

\section{Temporal analysis}

The epidemic curve (Fig. 6) and descriptive epidemiology suggest that this was a continuous commonsource outbreak. Many of the cases had visited Barrow on more than one day, making it difficult to ascribe a definitive incubation period for them. Further investigation of the data revealed a subgroup of 30 cases, for which there is reasonable certainty of the (one) date that they had visited Barrow town centre (Fig. 7), and therefore, had only been exposed to this source of infection on one occasion. Of these 30 cases [five culture-positive (one fatal), $24 \mathrm{UAg}$ positive and one seropositive], 24 were pneumonic. Twenty-nine cases reported onset dates. Of these, one case, who reported an incubation of 49 days, has been excluded from this incubation period analysis since the symptoms history was too unclear to be ascribed with utmost certainty (this case may actually have been a sporadic community case), thus leaving 


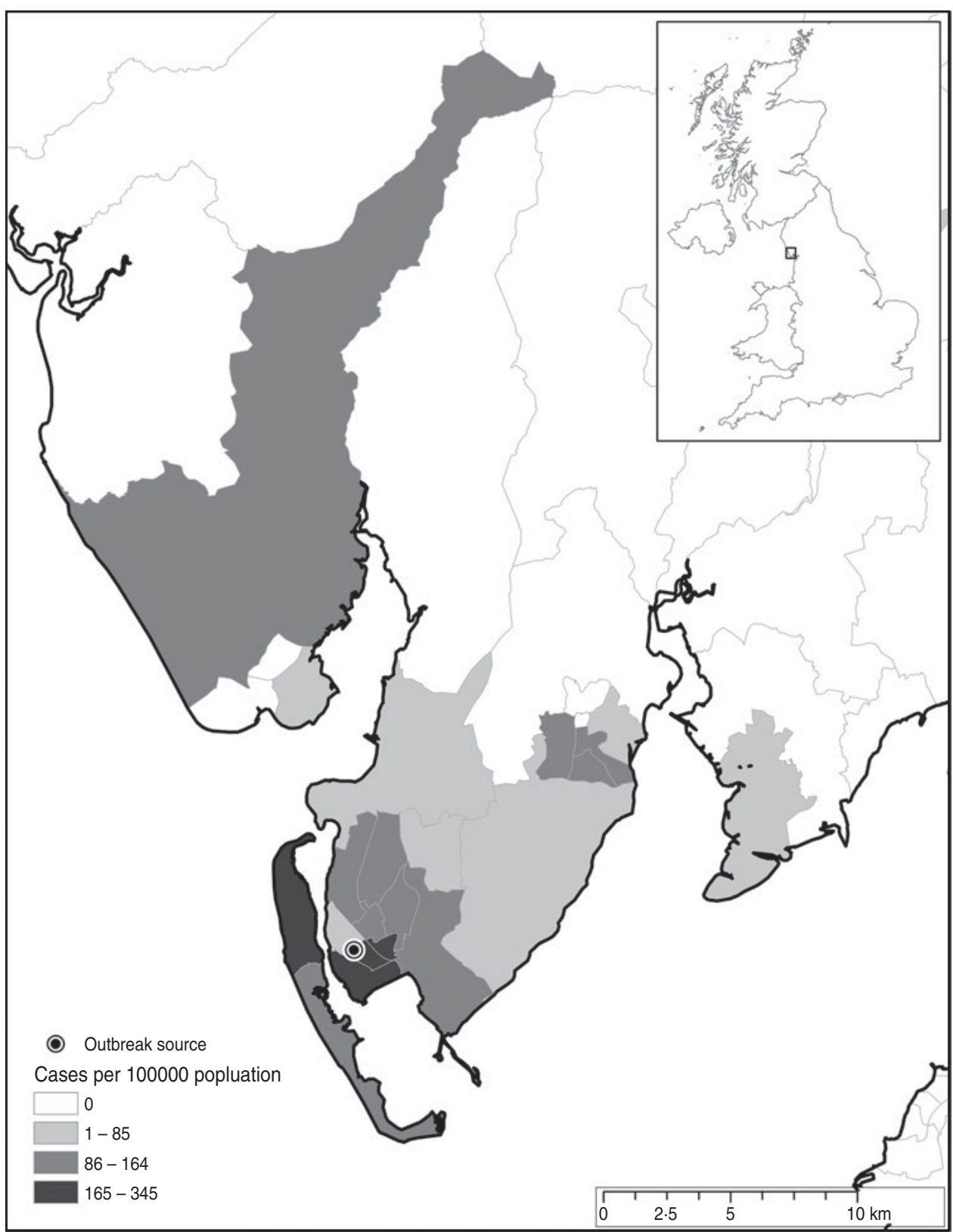

Fig. 5. Attack rate by home location, aggregated to Census Area Statistics (CAS) ward, Barrow-in-Furness.

28 cases. However, a case with a 34-day incubation had a very clear symptoms history and has therefore been included the analysis (Fig. 8). Data from these 28 cases give an incubation period range of between 1 and 34 days, (mean 7.1 days/median 6 days) (Fig. 8). Ages ranged between 40 and 86 years (mean 60.9/median 59.5 years); 15 were female. Four $(14 \cdot 3 \%)$ cases had an incubation period exceeding 10 days and three $(10 \cdot 7 \%)$ cases had an incubation of $<2$ days. These latter three were LD cases (one culture-positive and one each UAg-positive and seropositive). The dates that these 28 cases had been in Barrow town centre ranged from 27 June to 1 August, when the towers were shut down. This suggests that the cooling tower had been emitting infective aerosols from the middle to the end of June until the plant was switched off, but emitting the highest concentrations of infectious material mid-July (Fig. 7) when most people were infected. Using a recently published back-calculation method, we also estimated the period of exposure from the epidemic curve and prior knowledge of the incubation period distribution (based on 114 individual incubation periods obtained during an outbreak in Melbourne, 


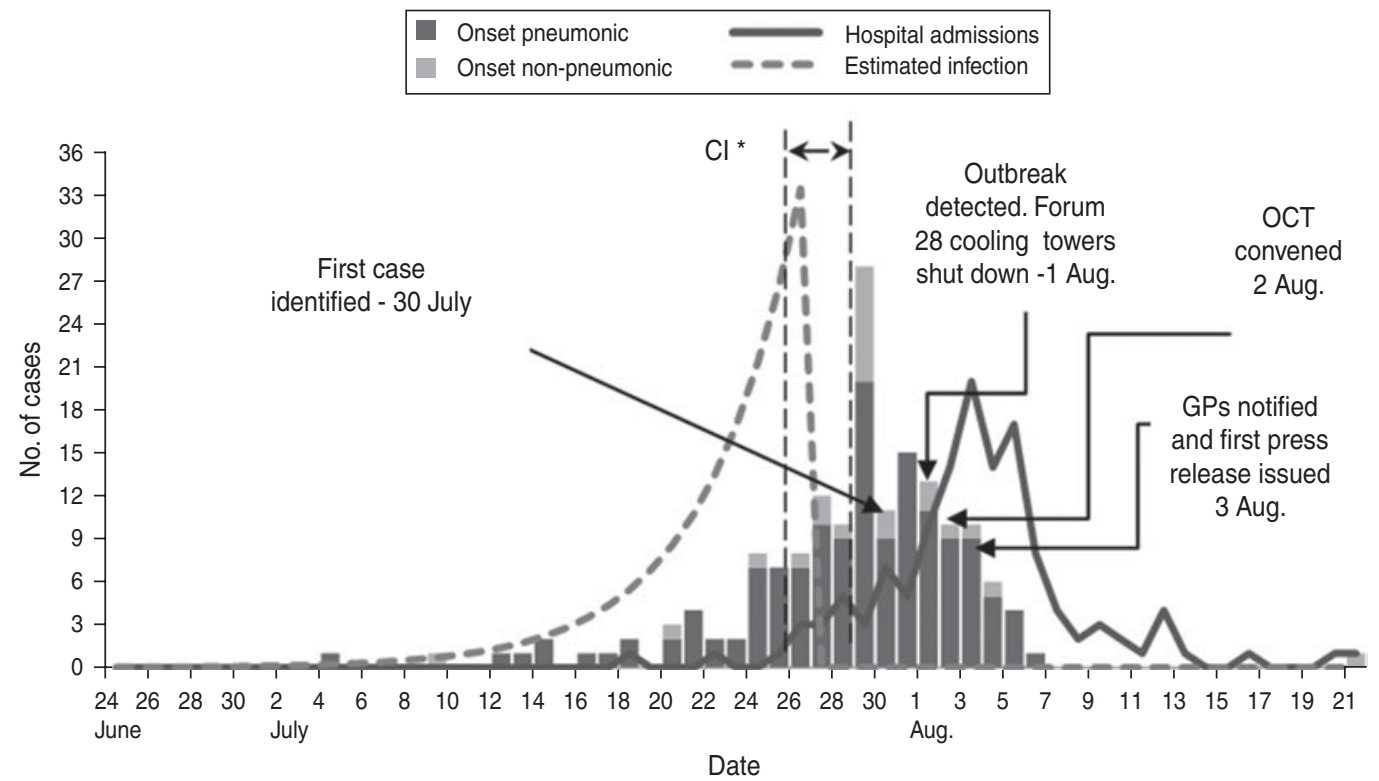

Fig. 6. Final epidemiological curve and estimated infection period. Number of legionellosis cases by date of onset of illness where known $(n=165)$ and date of hospital admission $(n=132)$, July to August 2002.

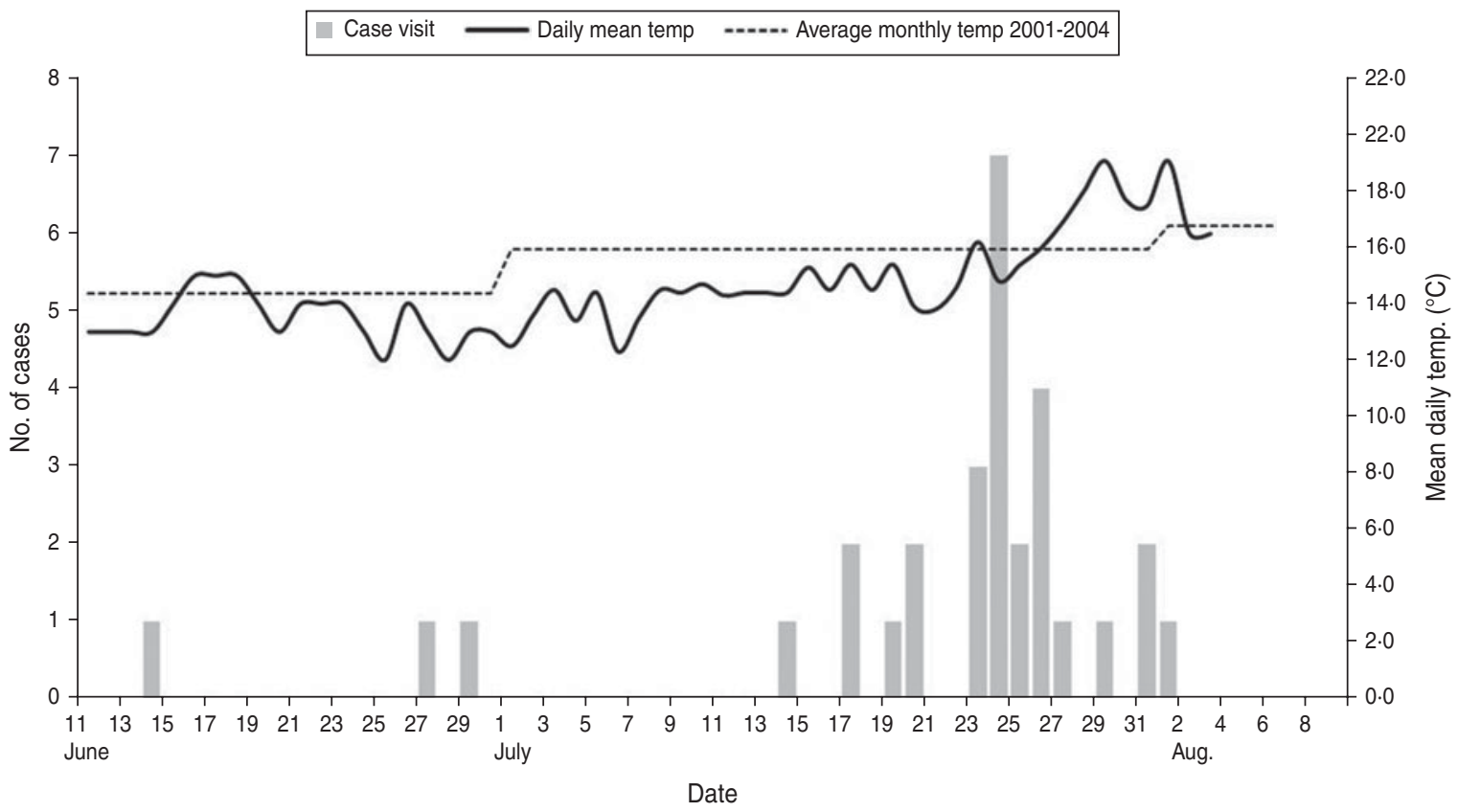

Fig. 7. Dates of case visits and daily mean temperature during likely period of emission of Legionella-contaminated aerosol, against average monthly temperature 2001-2004 $(n=28)$.

Australia) [19]. By assuming a release whereby the Legionella organisms are uniformly dispersed from the source with definite start and end times, with corresponding uniform infection in the exposed population, the modelled epidemic curve does not provide a close description of the observed symptom onset times $(P<0 \cdot 05)$. However, by assuming a slightly more complex release that infects an increasing number of people over time, to reflect the replication of Legionella organisms within the source, the modelled epidemic curve fits the data much better $(P=0 \cdot 17)$. Figure 6 shows the estimated number of infections 


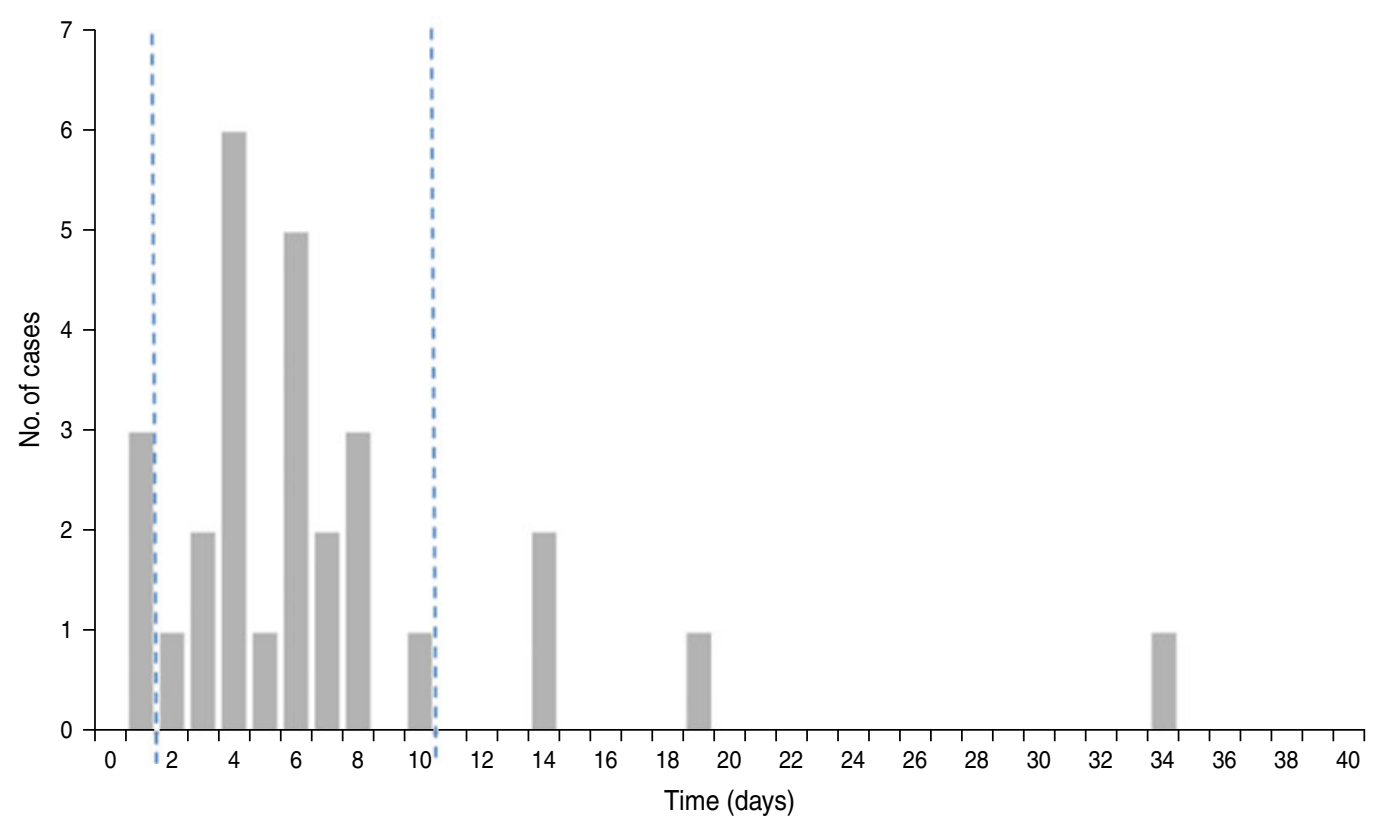

Fig. 8. Incubation period for subgroup of cases (one visit only) $(n=28)$. Number of cases with incubation period $<2$ days to $>7$ days $=7(25 \%)$. All confirmed Legionnaires' disease cases.

per day until the estimated end date of the release on 27 July (confidence interval 26-28 July). The reason the confidence interval does not capture the date the cooling tower was shut down (1 August) is because the incubation periods and infection times that occurred in this outbreak (see Figs 7 and 8) are unlikely to match exactly our idealized models. However, it is encouraging that such a parsimonious technique can estimate the end date of the release to within a few days of the actual closure of the source.

Twenty-two of the 28 cases included in the incubation period analysis were Barrow residents, with no reported history of travel outside Barrow. Three others resided in other parts of the country but stayed in Barrow for one or more nights during their incubation period and two other cases were non-Barrow residents but visited caravan sites local to Barrow as well as the town centre. The final case had unknown travel outside Barrow. As far as could be reasonably ascertained from direct questioning, these 28 cases were unlikely to have been exposed elsewhere, as no other outbreaks or clusters of legionellosis were identified in this region of the UK at that time. Interrogation of the National Surveillance Scheme database to find reports of sporadic cases near to their places of residences, confirmed to the authors that Barrow is the most likely source due to the number of cases in the area at the time of travel.
Forty percent (53/132) of all hospitalized cases (where full dates are known) were admitted before 3 August-when outbreak details were released to GPs and the public and 60\% (76/132) of hospitalized cases were admitted on or after 3 August. The period between onset of symptoms and hospitalization ranged from 0 to 32 days for 127 cases (where dates of both onset and admittance are known) (Fig. 9). The mean interval was 6 days (median 5 days), with a mean interval of 4.6 days for those admitted before 3 August and a mean interval of $7 \cdot 0$ days for those cases admitted on or after 3 August.

\section{Weather and environmental conditions}

The mean maximum daily temperature during July and the beginning of August was $17.7^{\circ} \mathrm{C}$ (range $14-21^{\circ} \mathrm{C}$ ). Figure 7 shows the daily mean temperature for the outbreak period compared to the average monthly temperatures for 2001-2004. June and July 2002 were not particularly hot months in the Barrow region, and July was not a particularly wet month with $58 \mathrm{~mm}$ of rainfall compared to a monthly average of $88 \mathrm{~mm}$ rainfall.

\section{Environmental investigation and microbiological study}

A rapid evaluation of the principal hazardous installations in the Barrow locality, together with evidence of 


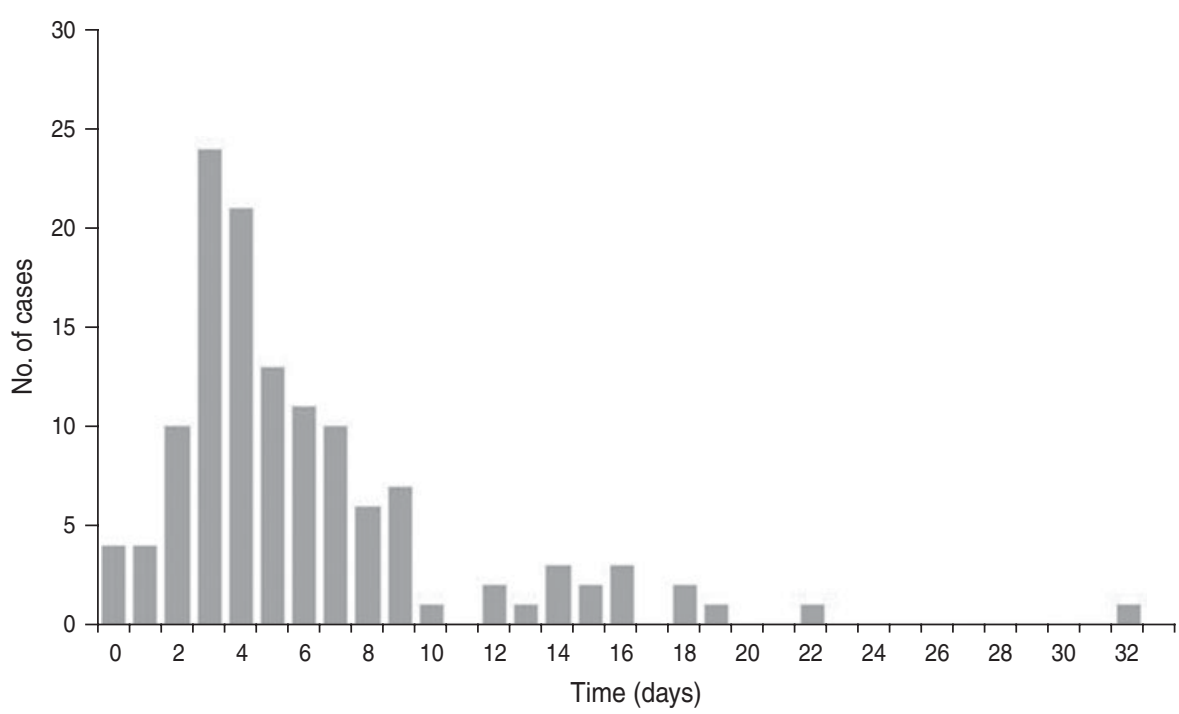

Fig. 9. Interval between onset of symptoms and admission to hospital $(n=127)$.

patients' movements, identified one probable suspect system - the forced-air water cooling tower for the air-conditioning unit at Forum 28. This unit was shut down the day before the OCT first met. Only four large-scale controlled wet-air conditioning systems are sited in the Barrow area, with the only one within the town centre itself being in the Forum 28 building. The site, owned and operated by Barrow Borough Council, is located in Barrow town centre with one side adjoining an alleyway, which is a main thoroughfare between shops and a number of bus stops (Fig. 2). In total, over 550 potentially hazardous premises in the Barrow area were inspected and sampled, where possible.

Legionellae were only recovered from two environmental sites: the air-conditioning unit of Forum 28 (two forced-air evaporative condensers, only one of which was in operation and was linked to an out-of-use tower which contained water) and a room in a Barrow retirement home, where L. pneumophila non-serogroup 1 were identified from hot and cold water systems serving a case's room. Only those bacteria grown from Forum 28 samples were of the strain most commonly associated with disease in humans $L$. pneumophila serogroup 1 -and the only strain identified in patients treated during the outbreak. A monoculture of $L$. pneumophila serogroup 1 was demonstrated by DFA in the pond water samples from the air-conditioning unit in counts that exceeded $10^{7}$ bacteria/litre water sampled. Subsequently, these environmental isolates were grown and epidemiological typing identified them as L. pneumophila serogroup 1, mAb2+ve subgroup Benidorm, AFLP type 033. Samples from other investigated airconditioning units showed no Legionella spp.

\section{Clinical microbiology}

Pneumonia was diagnosed, along with either a positive UAg or serology test, for 145 cases, 34 other test-positive cases of legionellosis without evidence of pneumonia were also detected (Fig. 4). Seventy-nine outbreak-related sputum samples were taken and L. pneumophila was isolated from 16 patients. Epidemiological typing showed all isolates to be L. pneumophila serogroup $1, \mathrm{mAb} 2+\mathrm{ve}$ subgroup 'Benidorm', AFLP type 033, hence indistinguishable from the isolates recovered from Forum 28 cooling tower's pond water. Subsequently, all isolates were examined by SBT and confirmed to be sequence type (ST)78.

\section{Police investigation}

All the evidence was managed by the police before passing the casework to the Crown Prosecution Service who progressed the legal case to court. The prosecution file ran to 3627 pages, over 200 witness statements were taken and over 200 exhibits in 10 crates were produced [20]. Two criminal court cases were held, the first in February 2005 charging Barrow Borough Council with corporate manslaughter-which was later dismissed, and the second case in June 2006 against a council employee, who was 
acquitted of manslaughter but charged under section 7 of the Health and Safety at Work Act 1974.

\section{DISCUSSION}

The large legionellosis outbreak in Barrow during the summer of 2002, illustrates the potential of wet airconditioning systems to rapidly infect a large number of people, if contaminated with Legionella. The source of exposure was a cooling tower of an air-conditioning plant at the Forum 28 arts and leisure centre, with all confirmed cases strongly linked to its vicinity. Early epidemiological and microbiological studies confirmed this association. Indeed, full molecular characterization of the clinical and environmental isolates was reported to the OCT within a week of the outbreak being recognized, providing further weight of evidence that Forum 28 was the outbreak source. Additionally, there has been no evidence of further cases in the local area being exposed to the outbreak strain following the shut down of the cooling towers.

The observation by the senior EHO of the drift emanating into the alleyway and his shutting down of the system, meant that the release of infectious aerosol was stopped, fortuitously, but coincidentally, on the same day as the outbreak was recognized. The EHO quickly instigated investigation of the hot and cold water systems at the arts and leisure centre and initiated wider investigation of all other registered (and unregistered) potentially hazardous installations in the town. A wet cooling systems register is a very valuable starting point for environmental investigation and can speed up the process of identification in an outbreak situation [21]. Indeed, it was following the Public Enquiries in response to the cooling towerassociated outbreaks at Stafford hospital [22] and the BBC [26] that regulations for registration were introduced in England, Wales and Scotland in the early 1990s. Other large outbreaks led to similar introductions in other European countries. Two days after the shut down, testing of water concentrates showed positive results of $\mathrm{mAb2}+\mathrm{ve}$ organisms at $\sim 10^{7}$ colony-forming units/litre sampled water. Urine results had already shown the outbreak strain to be L. pneumophila serogroup $1 \mathrm{mAb} 2+\mathrm{ve}$ and, since these strains are relatively unusual in environmental samples and were detected in such high numbers in the putative source, investigators were confident from this evidence that Forum 28 was the probable source-and consequently that the outbreak had been 'controlled' since the cooling towers had been shut down. Respiratory samples were found to be indistinguishable from bacteria grown from the cooling tower pond water samples: L. pneumophila serogroup 1, mAb2+ve Benidorm, AFLP type 033 (ST78), a strain which was at the time, and remains, unique in the European reference database of more than 1370 distinct sequence types. Further investigation of Forum 28 determined that only one of the two cooling towers was operational at the time of the outbreak, but was linked to the second, out-of-use tower, still containing water which provided areas of 'dead space' for contaminated water to accumulate. The two biocide barrels were empty and it has been estimated that biocide would not have been delivered into the system since December 2001, approximately 6 months before the outbreak [20]. The air-conditioning unit itself had not been maintained for around 12 months prior to the outbreak - no microbiological monitoring or system checks had been conducted due to a change in maintenance provision. The high bacterial counts in the unit were at levels that have given rise to outbreaks in other reported incidents and far exceed the corrective action level as laid down in HSE L8 guidelines [23]. After its shut down, the tower was decommissioned with two tonnes of heavily contaminated water being treated and discharged. Other large, potentially hazardous, plants in the Barrow area were shock-dosed with biocides in case of seeding from the Forum 28 plant, and their acting as a secondary outbreak source. Evidence that no new cases had been detected following the closure of the air-conditioning plant (outside of the known incubation period of legionellosis) and the fact that the towers were dismantled, rather than just being shut down, treated and reopened, allowed assurances to be made to the public (and investigators alike) that the source of infection had been correctly identified and controlled.

The epidemiological linkage between the first two cases, and the speed with which the public health response was initiated, was largely due to the newly integrated public health team structure in the North West of England, formed in the shadow of the nascent Health Protection Agency. The surveillance and internal communications systems within the unit undoubtedly contributed to a swift and effective response, with a large team of people with pertinent skills being immediately available. Technical support given by other agencies, including the Police and HSE, was another timely contributory factor to the efficient response to the outbreak. Also of significance 
Table 3. Case numbers, case-fatality rates and hospitalization rates of several large UK and European cooling tower-associated community legionellosis outbreaks

\begin{tabular}{|c|c|c|c|c|c|c|c|}
\hline Outbreak & Year & $\begin{array}{l}\text { Cases } \\
\text { (deaths) }\end{array}$ & $\begin{array}{l}\text { Case- } \\
\text { fatality } \\
\text { rate }(\%)\end{array}$ & $\begin{array}{l}\text { Hospitalized } \\
(n)\end{array}$ & $\begin{array}{l}\text { Hospitalized } \\
(\%)\end{array}$ & $\begin{array}{l}\text { Intensive care/ } \\
\text { mechanical } \\
\text { ventilation cases } \\
(n)\end{array}$ & $\begin{array}{l}\text { Intensive care/ } \\
\text { mechanical } \\
\text { ventilation } \\
(\%)\end{array}$ \\
\hline Stafford Hospital [22] & 1985 & $68(22)$ & $32 \cdot 0$ & 68 & $100 \cdot 0$ & - & - \\
\hline $\begin{array}{l}\text { Glasgow Royal } \\
\text { Infirmary [24] }\end{array}$ & 1985 & $16(5)$ & $31 \cdot 3$ & 16 & $100 \cdot 0$ & 4 & $25 \cdot 0$ \\
\hline Gloucester [25] & 1986 & $18(3)$ & $16 \cdot 6$ & 12 & $66 \cdot 6$ & - & - \\
\hline $\mathrm{BBC}[26]$ & 1988 & $79(3)$ & $3 \cdot 8$ & - & - & 11 & $14 \cdot 0$ \\
\hline Nottingham [27] & 1988 & $14(2)$ & $14 \cdot 0$ & 12 & $86 \cdot 0$ & 1 & $2 \cdot 9$ \\
\hline Piccadilly Circus [28] & 1989 & $33(5)$ & $15 \cdot 0$ & - & - & - & - \\
\hline Netherlands [3] & 1999 & $188(17)$ & $9 \cdot 0$ & 163 & $87 \cdot 0$ & 34 & $26 \cdot 0$ \\
\hline Melbourne [29] & 2000 & $125(4)$ & $3 \cdot 2$ & 95 & $76 \cdot 0$ & - & - \\
\hline Barrow-in-Furness [5] & 2002 & $179(7)$ & $3 \cdot 9$ & 134 & $74 \cdot 9$ & 18 & $10 \cdot 0$ \\
\hline Catalonia [30] & 2002 & $113(2)$ & $1 \cdot 8$ & 83 & $73 \cdot 5$ & - & - \\
\hline Hereford [31] & 2003 & $28(2)$ & $7 \cdot 1$ & 23 & $82 \cdot 0$ & - & - \\
\hline Murcia [32] & 2003 & $449(5)$ & $1 \cdot 0$ & 332 & $74 \cdot 0$ & - & - \\
\hline Pas-de-Calais [33] & 2003/04 & $86(18)$ & $21 \cdot 0$ & 84 & $98 \cdot 0$ & - & - \\
\hline Sarpsborg [34] & 2005 & $56(10)$ & $17 \cdot 9$ & - & - & - & - \\
\hline Pamplona [35] & 2006 & $146(0)$ & $0 \cdot 0$ & 76 & $52 \cdot 0$ & 7 & $5 \cdot 0$ \\
\hline Edinburgh [36] & 2012 & $61(4)$ & $6 \cdot 5$ & 49 & $80 \cdot 3$ & 22 & $44 \cdot 5$ \\
\hline
\end{tabular}

- , Not reported.

Hospitalized $(\%)=$ no. hospitalized/no. cases; intensive case/mechanical ventilation $(\%)=$ no. cases requiring intensive care or mechanical ventilation/no. cases hospitalized.

was the early detection of the Forum 28 cooling tower as a suspect source and its pre-emptive shut down, only 2 days after the first legionellosis case was detected. Improved case ascertainment and prompt recognition and management of the outbreak, most probably helped to realize a low case CFR $(3.9 \%$, $4.8 \%$ for pneumonic cases), which compares favourably with other large UK and European legionellosis outbreaks, where some (non-hospital-based) have seen CFRs of up to $21 \%$ (Table 3). In comparison with earlier large UK outbreaks in the 1980s [22, 24, $25,27]$, where UAg testing had not been widely used as a diagnostic method (routine use began in the late 1990s in the UK), employment of UAg testing in Barrow may have reduced the number of deaths, by enabling prompt administration of appropriate treatment. Increased awareness by clinicians and the general public may also have aided this low CFR [37].

The hospitalization rate $(74.9 \%)$ is similar to average rates reported in other large outbreaks $(\sim 75 \%)$ (Table 3). Thirteen percent of those hospitalized required intensive care or mechanical ventilation, putting a considerable strain on local services, as discussed elsewhere $[5,6]$. In both sexes, the attack rate increased with increasing age, with people aged $\geqslant 80$ years having the highest attack rate, a pattern which mirrors European infection rates [38]. The attack rate here was similar between the sexes, an observation that can often be seen within outbreak settings. This contrasts with the 3:1 male to female ratio [38] observed in sporadic cases, reflecting, perhaps, a male bias in sporadic cases due to the nature of occupation or behaviour.

From the subset of cases who visited Barrow town centre only once, and for whom we have pertinent data, three cases had an incubation of $<2$ days and four cases had an incubation period exceeding 10 days (14, 19, 34 days). These longer incubation periods have also been reported in other large outbreaks $[2,3,19,29]$ suggesting that it may be pragmatic to consider longer incubation periods in some outbreak case definitions. This is supported by actions taken by the Dutch public health authorities who use a 21-day limit in their incubation period following the 1999 outbreak in The Netherlands [39]. In the Barrow outbreak, the case with the 34-day incubation period (a 46-year-old, hospitalized with LD) only visited Barrow once during the investigation period and had no other known risk exposures, suggesting that the long incubation period has been accurately calculated. 
However, since this was not a culture-proven case, it cannot be excluded as a simultaneously detected sporadic case from elsewhere. The only way to determine the probable source of an infection with utmost certainty is by comparing clinical and environmental isolates using nucleic acid techniques. Culture for Legionella sp. is slow and hence of limited clinical utility, and due to the difficulty in obtaining and culturing respiratory samples, UAg testing is more readily used, particularly in large outbreak settings. Therefore, without a clinical isolate, we cannot say categorically that the source of infection for this case was Forum 28 , but there is strong epidemiological evidence that this case was part of the outbreak: they lived in the outbreak region, reported being in the close vicinity to Forum 28 and had no evidence of travel outside that region within the incubation period.

Three cases had an incubation period of $<2$ days. This may be because the reported short incubation periods were due to recall bias, i.e. some cases may have been more likely than others to report symptoms early on, depending on current or past health experiences, or a reporting bias because whole-day units are recorded. It is also possible that these incubations were genuinely short ( $<2$ days) and reflect an unknown dose dependency. The unusual circumstances of the exposure - in an enclosed alleyway - may have led to unusually sizable infective doses resulting, perhaps, in these short incubations.

It is clear that a relatively large number of cases became symptomatic before the outbreak was recognized on 1 August, and were only identified retrospectively through active case-finding. This is a feature often associated with outbreak investigation-the lead time between onset of symptoms and clinical evidence of disease means that, as in this outbreak, the first linkage of cases is often correlated with the peak of symptomatic cases in community outbreaks. However, a factor that may be of importance here is that in Barrow at that time, there was a high prevalence of chronic respiratory disease associated with smoking and asbestos [40, 41]. Asbestosis is a chronic long-term lung condition, caused by prolonged exposure to asbestos - a material historically used widely in heavy industries such as those associated with Barrow. This background of disease may have masked early clinical symptoms leading to the suspicion and investigation of legionellosis.

Weather conditions in Barrow were not especially favourable for the growth of Legionella at the time of the outbreak; daily temperatures ranged from $14^{\circ} \mathrm{C}$ to $21^{\circ} \mathrm{C}$ (naturally occurring L. pneumophila survive and multiply in water at temperatures between $25^{\circ} \mathrm{C}$ and $45^{\circ} \mathrm{C}$, with an optimal temperature range of $32-42^{\circ} \mathrm{C}$ [18]). The cause of the outbreak was the continued neglect of the maintenance of the airconditioning system which provided the opportunity for organisms to grow unchecked: 'over a 12-month period, failure to properly maintain the automatic dosing system and failure to identify that the system was not being maintained led directly to the tragic events in August 2002' [20]. Analysis of the epidemic curve suggests an exposure window that would result from the number of aerosolized organisms starting at very low levels but then exponentially increasing up until the end of July. These estimates correspond with the subgroup of individuals $(n=28)$ who were likely to have been exposed on only one occasion, most of whom were in Barrow town centre from mid-to-late July and the closure of the Forum 28 cooling tower at the beginning of August. The circumstances around the source of exposure are in themselves unusual, in that the contaminated aerosol would arguably have been contained for longer in the alleyway, as opposed to being dispersed and diluted into the open air; more concentrated doses may have contributed to the large number of cases. However, the timely recognition and management of the outbreak, and the greater proportion of mild cases detected with the employment of UAg testing, very likely led to the low CFR.

Inevitably, there were challenging aspects to this large outbreak investigation, particularly as it was conducted alongside a criminal investigation and a major incident response. These aspects have been discussed elsewhere [5,6]. Carrying out parallel examinations, with their own different cultures of investigation and processes, signalled a new phase of public health practice, and indeed this was the first time that outbreak team function and discussion had been noted as part of police evidence. The counterpoint to this was that public discussion about the detail of the outbreak had to take account of on-going legal proceedings, indeed the 10-year delay in publishing this report is, in large part, a consequence of this legal process.

From the outset, good cooperation and teamwork existed between all partners in the investigation, at local, regional and national levels. The early and sustained communication with health professionals, media and the local population was a very important part of this investigation and not only resulted in low community tension, but also helped to relieve pressure 
on the main hospital, enabling staff to meet the demands of caring for the higher than average number of admissions. This outbreak remains the largest legionellosis outbreak in the UK to date. However, its impact in the region was undoubtedly reduced due to the swift response and collaboration of all agencies involved. The incident does, nevertheless, add to the increasing body of evidence of cooling towers being a significant habitat of legionellae and highlights the inevitable and regrettable cost of a legionellosis outbreak to a community.

\section{ACKNOWLEDGEMENTS}

The authors acknowledge the contribution of Dr P. A. Wright to the outbreak investigation, particularly at the beginning of the outbreak where he played a key role in sample collection from the airconditioning plant in Forum 28. We thank all those individuals involved in the outbreak - local, regional and national Health Protection Agency staff, the Local Authority, the Health and Safety Executive, Environmental Health Officers, hospital staff and microbiologists - for their dedication and professionalism in the face of such a large outbreak. We also acknowledge the tragic loss of seven members of the public and recognize the effect of the incident on a large number of others.

\section{DECLARATION OF INTEREST}

None.

\section{REFERENCES}

1. Castilla J, et al. A large Legionnaires' disease outbreak in Pamplona, Spain: early detection, rapid control and no case fatality. Epidemiology and Infection 2008; 136: 823-832.

2. Okada M, et al. The largest outbreak of legionellosis in Japan associated with spa baths: epidemic curve and environmental investigation in Japanese. Kansenshogaku Zasshi 2005; 79: 365-374.

3. Den Boer JW, et al. A large outbreak of Legionnaires' disease at a flower show, the Netherlands, 1999. Emerging Infectious Diseases 2002; 8: 37-43.

4. De Schrijver K, et al. An outbreak of Legionnaires' disease among visitors to a fair in Belgium in 1999. Public Health 2003; 117: 117-124.

5. Telford D, et al. The legionnaires' outbreak in Barrow-in-Furness, summer 2002. Journal of Epidemiology and Community Health 2006; 60: 464-466.

6. Smith AF, Wild C, Law J. The Barrow-in-Furness legionnaires' outbreak: qualitative study of the hospital response and the role of the major incident plan. Emergency Medicine Journal 2005; 22: 251-255.

7. Anon. CDR Weekly, volume 12, number 32, 8 August 2002.

8. Anon. CDR Weekly, volume 12, number 33, 15 August 2002.

9. Birtles RJ, et al. Evaluation of urinary antigen ELISA for diagnosing Legionella pneumophila serogroup 1 infection. Journal of Clinical Pathology 1990; 43: 685-690.

10. Dournon E. Isolation of legionellae from clinical specimens. In: Harrison TG, Taylor AG, eds. A Laboratory Manual for Legionella. Chichester: John Wiley and Sons, 1988, pp. 13-30.

11. Helbig JH, et al. Pan-European study on culture-proven Legionnaires' disease: distribution of Legionella pneumophila serogroups and monoclonal subgroups. European Journal of Clinical Microbiology and Infectious Diseases 2002; 21: 710-716.

12. Fry NK, et al. Designation of European Working Group on Legionella Infection amplified fragmentlength polymorphism types of Legionella pneumophila serogroup 1 and results of intercentre proficiency testing using a standard protocol. European Journal of Clinical Microbiology and Infectious Diseases 2002; 21: 722-728.

13. Gaia V, et al. Sequence-based typing of Legionella pneumophila serogroup 1 offers the potential for true portability in legionellosis outbreak investigation. Journal of Clinical Microbiology 2003; 41: 2932-2939.

14. Ratzow S, et al. Addition of neuA, the gene encoding $\mathrm{N}$-acylneuraminate cytidylyl transferase, increases the discriminatory ability of the consensus sequence-based scheme for typing Legionella pneumophila serogroup 1 strains. Journal of Clinical Microbiology 2007; 45: 1965-1968.

15. Harrison TG, Taylor AG. The diagnosis of Legionnaires' disease by estimation of antibody levels. In: Harrison TG, Taylor AG, eds. A Laboratory Manual for Legionella. Chichester: John Wiley and Sons, 1988, pp. 113-136.

16. British Standards Institution. BS 6068-ISO 11731 Water Quality-Part 4: Microbiological methods-Section 4.12: 1998 detection and enumeration of Legionella. London: British Standards Institution, 1998.

17. Harrison TG, Taylor AG. Demonstration of legionellae in clinical specimens. In: Harrison TG, Taylor AG, eds. A Laboratory Manual for Legionella. Chichester: John Wiley and Sons, 1988, pp. 103-112.

18. Bartram J, et al. (eds). Legionella and the prevention of legionellosis. Geneva, Switzerland: World Health Organisation, 2007.

19. Egan JR, et al. Modelling Legionnaires' disease outbreaks: estimating the timing of an aerosolized release using symptom-onset dates. Epidemiology 2011; March, 22.

20. Health \& Safety Executive. Report of the public meetings into the Legionella outbreak in Barrow-in-Furness, August 2002. Health and Safety Executive, 2007.

21. Ricketts KD, et al. Survey on legislation regarding wet cooling systems in European countries. Eurosurveillance 2008; 13: 18982 . 
22. O'Mahony M, et al. The Stafford outbreak of Legionnaires' disease. Epidemiology and Infection 1990; 104: 361-380.

23. Anon. Legionnaires' disease. The control of Legionella bacteria in water systems. Approved Code of Practice \& Guidance L8 Health and Safety Executive, 2000 (http://www.hse.gov.uk/pubns/priced/18.pdf). Accessed 10 April 2013.

24. Winter JH, et al. Rapid diagnosis of an outbreak of Legionnaires' disease at Glasgow Royal Infirmary. Thorax 1987; 42: 596-599.

25. Hunt DA, et al. An outbreak of Legionnaires' disease in Gloucester. Epidemiology and Infection 1991; 107: 133-141.

26. Broadcasting House Legionnaires' disease. Westminster Action Committee. London: City of Westminster, 1988.

27. Colville A, et al. Outbreak of Legionnaires' disease at University Hospital, Nottingham. Epidemiology, microbiology and control. Epidemiology and Infection 1993; 110: $105-116$.

28. Watson JM, et al. Piccadilly Circus Legionnaires' disease outbreak. Journal of Public Health 1994; 16: 341-347.

29. Greig JE, et al. An outbreak of Legionnaires' disease at the Melbourne Aquarium, April 2000: investigation and case-control studies. Medical Journal of Australia 2004; 180: $566-572$.

30. Sabria M, et al. A community outbreak of Legionnaires' disease: evidence of a cooling tower as the source. Clinical Microbiology and Infection 2006; 12: 642-647.

31. Kirrage D, et al. Investigation of an outbreak of Legionnaires' disease: Hereford, UK 2003. Respiratory Medicine 2007; 101: 1639-1644.

32. Garcia-Fulgueiras A, et al. Legionnaires' disease outbreak in Murcia, Spain. Emerging Infectious Diseases 2003; 9: 915-921.

33. Nguyen TMN, et al. A community-wide outbreak of Legionnaires disease linked to industrial cooling towers-how far can contaminated aerosols spread? Journal of Infectious Diseases 2006; 193: 102-11.

34. Nygård K, et al. An outbreak of Legionnaires' disease caused by long-distance spread from an industrial air scrubber in Sarpsborg, Norway. Clinical Infectious Diseases 2008; 46: 61-69.

35. Castellani-Pastoris M, et al. Molecular epidemiology of an outbreak of Legionnaires' disease associated with a cooling tower in Genova-Sestri Ponente, Italy. European Journal of Clinical Microbiology and Infectious Diseases 1997; 16: 883-892.

36. Irons JF, et al. The effect of a large Legionnaires' disease outbreak in Southwest Edinburgh on acute and critical care services. QJM: an International Journal of Medicine. Published online: 22 August 2013. doi:10.1093/qjmed/hct167.

37. Joseph CA, Ricketts KD, on behalf of the European Working Group for Legionella Infections. Legionnaires' disease in Europe 2007-2008. Eurosurveillance 2010; 15: 19493

38. European Centre for Disease Prevention and Control. Legionnaires' disease in Europe 2009. Stockholm: ECDC, 2011 (http://ecdc.europa.eu/en/publications/ Publications/1109_SR_Legionnaires\%27\%20disease_ Europe_2009.pdf). Accessed November 2012.

39. Sonder GJ, et al. Changes in prevention and outbreak management of Legionnaires' disease in the Netherlands between two large outbreaks in 1999 and 2006. Eurosurveillance 2008; 13: 38.

40. McElvenny D, et al. Mesothelioma mortality in Great Britain from 1968 to 2001. Occupational Medicine 2005; 55: 79-87.

41. NHS Cumbria. Baseline Health Assessment for Barrowin-Furness: 2009 (http://www.barrowbc.gov.uk/pdf/ Baseline $\% 20$ Health $\% 20$ Assessment $\% 20$ for $\% 20$ Barrowin-Furness\%202009.pdf). Accessed April 2013. 